International Journal of Pure and Applied Mathematics

Volume 114 No. 1 2017, 105-111

ISSN: 1311-8080 (printed version); ISSN: 1314-3395 (on-line version)

url: http://www.ijpam.eu

doi: 10.12732 /ijpam.v114i1.9

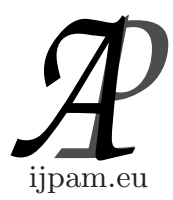

\title{
ON SPECTRA OF GLUED COMPLETE GRAPHS
}

\author{
C.Y. Jung ${ }^{1}$, A.R. Nizami ${ }^{2}$, M. Munir ${ }^{3}$, W. Nazeer ${ }^{4}$, S.M. Kang ${ }^{5} \S$ \\ ${ }^{1}$ Department of Business Administration \\ Gyeongsang National University \\ Jinju, 52828, KOREA \\ ${ }^{2,3,4}$ Department of Mathematics and Statistics \\ University of Lahore \\ Lahore, 54000, PAKISTAN \\ ${ }^{5}$ Department of Mathematics and RINS \\ Gyeongsang National University \\ Jinju, 52828, KOREA
}

\begin{abstract}
In this article, we give spectra and characteristic polynomial of three partite complete graphs. We also give spectra of cartesian and tenor product of $K_{\mathrm{n}, \mathrm{n}, \mathrm{n}}$ with itself. Finally, we give general closed forms of the characteristic polynomials of the graphs obtained by identifying two copies of $K_{\mathrm{n}}$ at a vertex and an edge.
\end{abstract}

AMS Subject Classification: $05 \mathrm{C} 12$

Key Words: characteristic polynomial, eigenvalue, spectrum

\section{Introduction}

Spectral graph theory is the study of algebraic properties of matrices associated with graphs. In particular, it is the study of spectra of adjacency matrices of graphs.

Received: $\quad$ February 2, 2017

Revised: $\quad$ March 22, 2017

Published: $\quad$ April 20, 2017

(c) 2017 Academic Publications, Ltd. url: www.acadpubl.eu

${ }^{\S}$ Correspondence author 


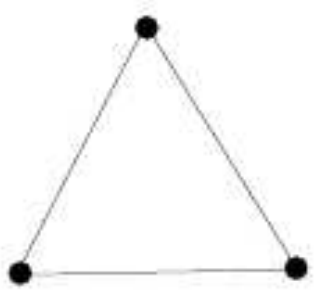

$\mathrm{K}_{3}$
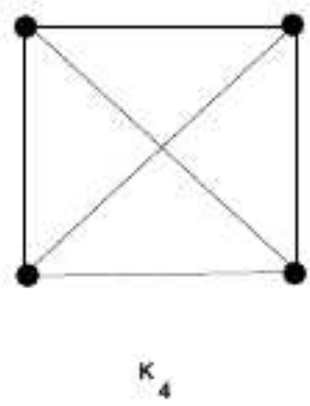

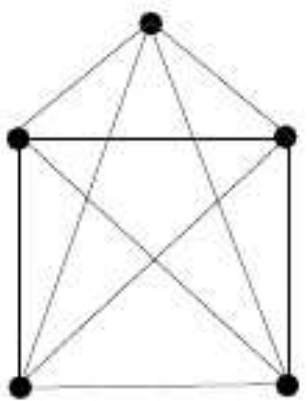

$\mathrm{K}_{5}$

Figure 1. Complete graphs

Spectral graph theory has several important applications in computer science, differential geometry, Riemannian geometry, Markov chains, and astronomy. The founder of Google computed Perron-Frobenius eigenvector of the web graph, and thus applied it in computer sciences, see [9]. The second largest eigenvalue is used in statistics to gain information about expansion and randomness properties of graphs. The smallest eigenvalue is important for chromaticity. For details, see $[13,14,17]$.

A graph $G$ is a pair $(V, G)$, where $V$ is the set of vertices and $E$ is the set of edges. The adjacency matrix associated to a finite connected graph $G$ on vertices $n$ is the $n \times n$ matrix $A=\left\{a_{i j}\right\}$, where $a_{i j}$ is the number of edges between the vertices $i$ and $j$. As an example, you can see the adjacency matrices of the complete graphs $K_{3}, K_{4}$ and $K_{5}$ :

$$
A_{K_{3}}=\left(\begin{array}{ccc}
0 & 1 & 1 \\
1 & 0 & 1 \\
1 & 1 & 0
\end{array}\right) ; A_{K_{4}}=\left(\begin{array}{cccc}
0 & 1 & 1 & 1 \\
1 & 0 & 1 & 1 \\
1 & 1 & 0 & 1 \\
1 & 1 & 1 & 0
\end{array}\right) ; A_{k_{5}}=\left(\begin{array}{lllll}
0 & 1 & 1 & 1 & 1 \\
1 & 0 & 1 & 1 & 1 \\
1 & 1 & 0 & 1 & 1 \\
1 & 1 & 1 & 0 & 1 \\
1 & 1 & 1 & 1 & 0
\end{array}\right)
$$

A real number $\lambda$ is said to be an eigenvalue of an $n \times n$ matrix $A$ if there exist a nonzero vector $x$ of $\mathbb{R}^{n}$ such that $\lambda x=A x$ and $x$ is called the eigenvector corresponding to $\lambda$. Eigenvalues are usually computed as the roots of the characteristic polynomial $\Delta(A)=\left|\lambda I_{n}-A\right|$. The spectrum of a graph $G$ is the 

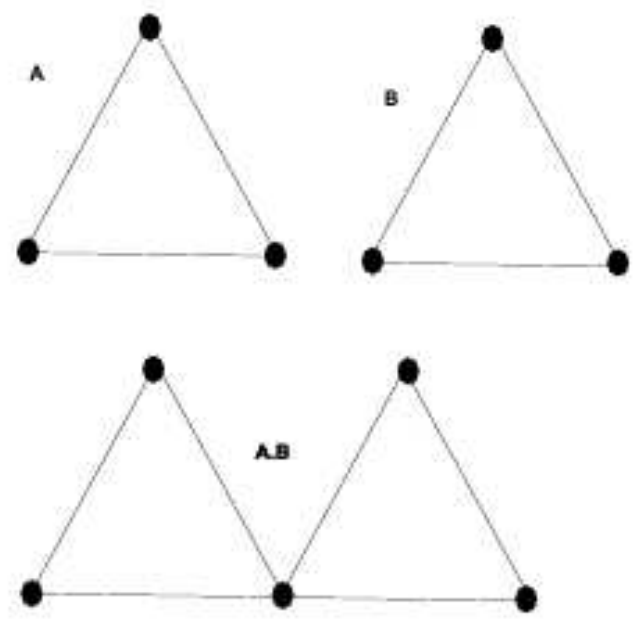

Figure 2. Vertex identification

collection of eigenvalues along with their multiplicities of the adjacency matrix of $G$.

Before going further, for some notations, by the graph $G_{1} \cdot G_{2}$ we shall mean the identification of a vertex of $G_{1}$ with a vertex of $G_{2}$, and by the graph $G_{1} \mathrm{I} G_{2}$ we shall mean the identification of an edge of $G_{1}$ with an edge of $G_{2}$.

\section{Main results}

This section contains the main results, including the characteristic polynomial of $K_{n, n, n}$, spectra of the cartesian and tenor products of $K_{n, n, n}$ with itself, and the closed forms of the characteristic polynomials of $K_{n} \cdot K_{n}$ and $K_{n} \mathrm{I} K_{n}$.

Theorem 2.1. The characteristic polynomial of $K_{n, n, n}$ is

$$
\lambda^{3 n-3}(\lambda+n)^{2}(\lambda-2 n)
$$

Proof. The adjacency matrix of $K_{n, n, n}$ is the $3 n \times 3 n$ matrix

$$
A_{K \mathrm{n}, \mathrm{n}, \mathrm{n}}=\left(\begin{array}{ccc}
O & J & J \\
J & O & J \\
J & J & O
\end{array}\right),
$$

where $J$ is the $n \times n$ matrix of 1 s and $O_{n}$ is the null matrix of order $n$. In the lower upper factorization of $\left(A_{K_{\mathrm{n}, \mathrm{n}, \mathrm{n}}}-\lambda I_{3 n}\right)$, the determinant of the main 


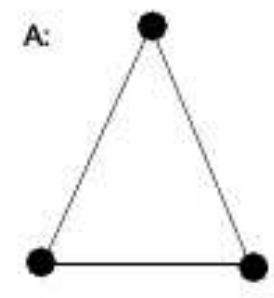

B:
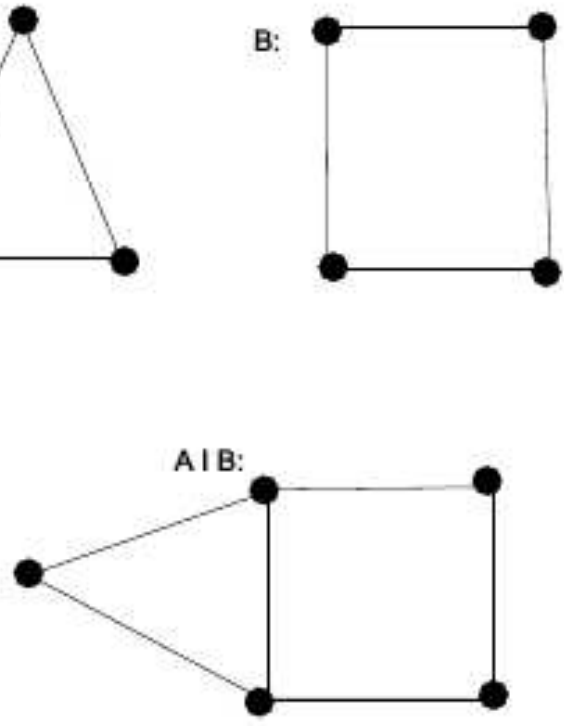

Figure 3. Edge identification

diagonal of lower triangular matrix $L$ is 1 as $\operatorname{diag}(L)=(1,1,1, \ldots, 1)$, and the determinant of the upper triangular matrix $U$ is

$$
\begin{aligned}
\operatorname{det}(U)= & \lambda^{n}\left(\frac{\lambda^{2}-n}{\lambda}\right)\left(\frac{\lambda^{2}-n \lambda-2 n}{\lambda-n}\right) \\
& \times\left(\prod_{m=1}^{n-1} \frac{A+(m-1) C}{B+(m-1) D}, n \geqslant 2\right) \\
& \times\left(\prod_{m=1}^{n-1} \frac{E+(m-1) G}{F+(m-1) H}, n \geqslant 2\right),
\end{aligned}
$$

where

$$
\begin{aligned}
& A=\left(\lambda^{2}-2 n\right) \lambda, \quad B=\lambda^{2}-n, \\
& C=-n \lambda, \quad D=-n, \\
& E=\left(\lambda^{2}-n \lambda-4 n\right) \lambda, \quad F=\lambda^{2}-n \lambda-2 n, \\
& G=-2 n \lambda, \quad H=-2 n .
\end{aligned}
$$

Now, by simplifying, we get the desire result.

Following Theorem 2.1 and [8] we get the following corollaries: 
Corollary 2.2. The spectrum of $K_{m, m, m}^{* 2}$ is

$$
\left(\begin{array}{cccccc}
-2 m & -m & 0 & m & 2 m & 4 m \\
4 & 12(m-1) & (m-1) & 4 & 6(m-1) & 1
\end{array}\right)
$$

Corollary 2.3. The spectrum of $K_{m, m, m}^{\otimes 2}$ is

$$
\left(\begin{array}{cccc}
-2 m^{2} & 0 & m^{2} & (2 m)^{2} \\
4 & 9\left(m^{2}-1\right) & 1 & 1
\end{array}\right)
$$

Theorem 2.4. The characteristic polynomial of $K_{n} \cdot K_{n}, n \geq 3$, is

$$
(\lambda+1)^{2 n-4}(\lambda-(n-2))\left(\lambda^{2}-(n-2) \lambda-2(n-1)\right) .
$$

Proof. The adjacency matrix of $K_{n} \cdot K_{n}$ is the $(2 n-1) \times(2 n-1)$ matrix

$$
A_{K_{\mathrm{n}} \cdot K_{\mathrm{n}}}=\left(\begin{array}{ccc}
0 & J & J \\
J^{T} & A_{K_{\mathrm{n}-1}} & O_{n-1} \\
J^{T} & O_{n-1} & A_{K_{\mathrm{n}-1}}
\end{array}\right)
$$

where $J$ is the $1 \times(n-1)$ matrix of $1 \mathrm{~s}$, and $A_{K_{\mathrm{n}-1}}$ is the adjacency matrix of $K_{n-1}$. Here $O$ is the null matrix. In the lower upper factorization of $\left(A_{K_{\mathrm{n}} \cdot K_{\mathrm{n}}}-\right.$ $\left.\lambda I_{2 n-1}\right)$, the determinant of the main diagonal of the lower triangular matrix $L$ is 1 as $\operatorname{diag}(L)=(1,1,1, \ldots, 1)$, and the determinant of the upper triangular matrix $U$ is

$$
\begin{aligned}
\operatorname{det}(U)= & \lambda\left(\frac{\lambda^{2}-1}{\lambda}\right)\left(\prod_{m=3}^{n} \frac{\lambda^{2}-(m-2) \lambda-(m-1)}{\lambda-(m-2)}\right) \\
& \times\left(\frac{\lambda^{3}-(n-2) \lambda^{2}-n \lambda+(n-2)}{\lambda^{2}-(n-2) \lambda-(n-1)}\right)\left(\prod_{m=1}^{n-2} \frac{C+(m-1) A}{D+(m-1) B}\right),
\end{aligned}
$$

where

$$
\begin{aligned}
& A=-\lambda^{3}+(n-4) \lambda^{2}+(3 n-6) \lambda+(2 n-3), \\
& B=-\lambda^{2}+(n-3) \lambda+2 n-3, \\
& C=\lambda^{4}-(n-2) \lambda^{3}-(n+2) \lambda^{2}+(3 n-8) \lambda+(3 n-5), \\
& D=\lambda^{3}-(n-2) \lambda^{2}-n \lambda+(n-2) .
\end{aligned}
$$

The result now follows from simple computations.

Theorem 2.5. The characteristic polynomial of $K_{n} \mathrm{I} K_{n}, n \geq 4$, is

$$
(\lambda+1)^{2 n-5}(\lambda-(n-3))\left(\lambda^{2}-(n-2) \lambda+(-3 n+5)\right) .
$$


Proof. The adjacency matrix of $K_{n} \mathrm{I} K_{n}$ is the $(2 n-2) \times(2 n-2)$ matrix

$$
A_{K_{\mathrm{n}} I K_{\mathrm{n}}}=\left(\begin{array}{cc}
A_{K_{\mathrm{n}}} & S_{(n-2, n)}^{T} \\
S_{(n-2, n)} & A_{K_{\mathrm{n}-2}}
\end{array}\right),
$$

where $A_{K_{\mathrm{n}}}$ is the adjacency matrix of $K_{n}$ and $S_{(n-2, n)}$ is $(n-2) \times n$ matrix

$$
S_{(n-2, n)}=\left(J_{(n-2 \times 2)} \quad O_{(n-2 \times n-2)}\right) .
$$

In the lower upper factorization of $\left(A_{K_{\mathrm{n}} I K_{\mathrm{n}}}-\lambda I_{2 n-2}\right)$, the determinant of the main diagonal of the lower triangular matrix $L$ is 1 as $\operatorname{diag}(L)=(1,1,1, \ldots, 1)$, and the determinant of the upper triangular matrix $U$ is

$$
\begin{aligned}
\operatorname{det}(U)= & \lambda\left(\frac{\lambda^{2}-1}{\lambda}\right)\left(\prod_{m=3}^{n} \frac{\lambda^{2}-(m-2) \lambda-(m-1)}{\lambda-(m-2)}\right) \\
& \times\left(\frac{\lambda^{3}-(n-2) \lambda^{2}-(n+1) \lambda+2 n-6}{\lambda^{2}-(n-2) \lambda-(n-1)}\right) \\
& \times\left(\prod_{m=1}^{n-3} \frac{C+(m-1) A}{D+(m-1) B}\right),
\end{aligned}
$$

where

$$
\begin{aligned}
& A=\lambda^{3}+(n-5) \lambda^{2}+(4 n-11) \lambda+3 n-8, \\
& B=\lambda^{2}+\lambda-(3 n+8) \\
& C=\lambda^{4}-(n-2) \lambda^{3}-(n+4) \lambda^{2}+(5 n-18) \lambda+(5 n-13), \\
& D=\lambda^{3}-(n-2) \lambda^{2}-(n+1) \lambda+(n-2) .
\end{aligned}
$$

This completes the proof.

\section{Conclusions}

In this article, we gave spectra and characteristic polynomials of three partite complete graphs using techniques of linear algebra and computer softwares. Technically speaking, we computed general closed forms of characteristic polynomials of the graphs obtained by gluing a vertex of $K_{n}$ with a vertex of another copy of $K_{n}$, and gluing an edge of $K_{n}$ with an edge of another copy of $K_{n}$. 


\section{References}

[1] O. Ahmadi, N. Alon, I.F. Blake, I.E. Shparlinski, Graphs with integral spectrum, Linear Algebra Appl. 430 (2009), 547-552 doi: 10.1016/j.laa.2008.08.020.

[2] N. Alon, Eigenvalues and expanders, Theory of computing (Singer Island, Fla., 1984), Combinatorica 6 (1986), 83-96, doi: 10.1007/BF02579166.

[3] S. Axler, Linear Algebra Done Right, Second edition, Springer-Verlag, New York (1997).

[4] H. Bai, The Grone-Merris Conjecture, arXiv:0911.2172 [math.CO].

[5] E. Bannai, T. Ito, On finite Moore graphs, J. Fac. Sci. Univ. Tokyo Sect. IA Math. 20 (1973), 191-208.

[6] E. Bannai, T. Ito, Algebraic Combinatorics I, Association Schemes, The Benjamin/Cummings Publishing Co., Inc., Menlo Park, CA (1984).

[7] B. Bollobás, Modern Graph Theory, Graduate Texts in Mathematics, Springer-Verlag, New York, (1998).

[8] A.E. Brouwer, W.H. Haemers, Spectra of Graphs, Springer, New York, (2012).

[9] K. Bryan, T. Leise, The $\$ 25,000,000,000$ eigenvector: the linear algebra behind Google, SIAM Rev. 48 (2006), 569-581, doi: 10.1137/050623280.

[10] H. Chuang, G.R. Omidi, Graphs with three distinct eigenvalues and largest eigenvalues less than 8, Linear Algebra Appl. 430 (2009), 2053-2062, doi: 10.1016/j.laa.2008.11.028.

[11] F.R.K. Chung, Spectral Graph Theory, CBMS Regional Conference Series in Mathematics, 92. Published for the Conference Board of the Mathematical Sciences, Washington, DC; by the American Mathematical Society, Providence, RI, (1997).

[12] S.M. Cioabă, Perfect matchings, eigenvalues and expansion, C. R. Math. Acad. Sci. Soc. R. Can. 27 (2005), 101-104.

[13] D.M. Cvetković, Graphs and their spectra, Univ. Beograd. Publ. Elektrotehn. Fak. Ser. Mat. Fiz. 354-356 (1971), 1-50.

[14] D.M. Cvetković, M. Doob, H. Sachs, Spectra of Graphs, Theory and Applications, Third edition, Johann Ambrosius Barth, Heidelberg, (1995).

[15] C.D. Godsil, Spectra of trees, Convexity and graph theory (Jerusalem, 1981), NorthHolland Math. Stud. 87 (1984), 151-159, Ann. Discrete Math., 20, doi: 10.1016/S03040208(08)72819-6.

[16] I. Gutman, P.J. Plath, On molecular graphs and digraphs of annulenes and their spectra, J. Serb. Chem. Soc. 66 (2001), 237-241.

[17] M. Petri, Spectral properties of generalized direct product of graphs, Ph.D. Thesis, University of Novi Sad, (1994).

[18] Ph. Delsarte, An algebraic approach to the association schemes of coding theory, Philips Res. Rep. Suppl. 10 (1973), 163-170. 
